\title{
Comparative genomic analysis of male and female poplars
}

\author{
Elena Pushkova \\ Engelhardt Institute of Molecular \\ Biology, RAS, Moscow, Russia \\ pushkova18@gmail.com \\ Nadezhda Bolsheva \\ Engelhardt Institute of Molecular \\ Biology, RAS, Moscow, Russia \\ nlbolsheva@mail.ru
}

\author{
George Krasnov \\ Engelhardt Institute of Molecular \\ Biology, RAS, Moscow, Russia \\ gskrasnov@mail.ru \\ Nataliya Melnikova \\ Engelhardt Institute of Molecular \\ Biology, RAS, Moscow, Russia \\ mnv-4529264@yandex.ru
}

\author{
Roman Novakovskiy \\ Engelhardt Institute of Molecular \\ Biology, RAS, Moscow, Russia \\ 0legovich46@mail.ru \\ Alexey Dmitriev \\ Engelhardt Institute of Molecular \\ Biology, RAS, Moscow, Russia \\ alex_245@mail.ru
}

\begin{abstract}
In the genomic analysis of trees, species belonging to the Populus genera serve as model objects. In our work, we performed genome sequencing of male and female plants of Populus $\mathbf{x}$ sibirica that is widespread in the Moscow region. To achieve high-quality de novo assemblies, we used a combination of two platforms, namely Oxford Nanopore and Illumina for obtaining long reads and high-accuracy short reads, respectively. In total, using a MinION sequencer, we obtained $18 \mathrm{~Gb}$ with average N50 of $25 \mathrm{~kb}$ for each genotype, whereas, using a HiSeq 2500 instrument, from 36 to 52 million pair-end $125+125$ reads were obtained for male and female plants. The Shasta assembler provided the best results for the assemblies: the $\mathrm{N50}$ values were about $0.5 \mathrm{Mb}$ and the integrity was over $95 \%$ according to BUSCO. The analysis of highquality genomes of male and female $P$. x sibirica trees provides an opportunity to reveal the genes associated with poplar sex that will facilitate the specification of its sex-determination mechanism.
\end{abstract}

Keywords - Populus, de novo genome assembly, Nanopore, Illumina, pure high-molecular-weight DNA

Motivation and Aim

\section{Motivation}

Poplar is a model object for genetic studies in trees [1]. In recent studies on the genetics of sex of the genus Populus, associated with sex polymorphisms were found and the areas of their localization in the genome were identified. However, many issues related to sex determination in poplar species have not yet been resolved, including the identification of the key genes and mechanisms that are involved in sex determination. $P . \times$ sibirica is one of the most common poplars in the Moscow region, but to date, there is no reference genome assembly for it. High-quality genome assemblies of male and female plants of $P$. x sibirica provide the opportunity to compare the results with previous studies of genetically different poplars and to suggest the mechanism of its sex determination.

Aim

This work aims to obtain high-quality assemblies of the genomes of male and female trees of $P$. $\times$ sibirica using a combination of Oxford Nanopore platform with long reads and Illumina with high-accuracy reads for further identification of genes that play a key role in determining the sex of poplar plants and to reveal the molecular mechanisms of their action.

\begin{abstract}
Methods
Plant material

Leaves were collected from male and female trees of $P . \mathrm{x}$ sibirica grown in different areas of Moscow. Young half-open leaves were gathered in the period of poplar bloom for the proper sex identification.
\end{abstract}

\section{DNA extraction}

For DNA extraction and purification, $P$. x sibirica leaves with a mass of $0.2 \mathrm{~g}$ were triturated in the mortar with liquid nitrogen. Then, we added $4 \mathrm{ml}$ of the Carlson lysis buffer (100 mM Tris-HCl pH 9.5 (VWR Life Science, USA); 2\% CTAB (VWR Life Science); 1.4 M NaCl (Scharlab, Spain); 1\% PEG 8000 (PanReac AppliChem, Germany); $20 \mathrm{mM}$ EDTA (Promega, USA)) that was prewarmed to $65^{\circ} \mathrm{C}$ and supplemented with $12 \mu$ of $\beta$-mercaptoethanol (BioRad, USA) and $0.04 \mathrm{~g}$ of PVP K30 (PanReac AppliChem). The homogenate was incubated in a "Gnom" thermostat (DNA Technology, Russia) at $65^{\circ} \mathrm{C}$ for 1 hour, with stirring every 10 min. Next, an equal volume of chloroform (Acros Organics, USA) was added to the homogenate, stirred on a Thermolyne Maxi Mix III Type 65800 shaker (Thermo Fisher Scientific, USA) at $800 \mathrm{rpm}$ for $10 \mathrm{~min}$, followed by centrifugation on a 5418R microcentrifuge (Eppendorf, Germany) using the following parameters: $10000 \mathrm{~g}, 10 \mathrm{~min}, 40^{\circ} \mathrm{C}$. The aqueous phase was transferred to a clean tube with the addition of 0.2 volume of 5x CTAB buffer (5\% CTAB, $350 \mathrm{mM}$ EDTA) and incubated at $65^{\circ} \mathrm{C}$ for $10 \mathrm{~min}$. After that, an equal volume of chloroform was introduced, stirred on a shaker for $10 \mathrm{~min}$, and centrifuged with the following parameters: $10000 \mathrm{~g}, 10 \mathrm{~min}$, $40^{\circ} \mathrm{C}$. The aqueous phase was transferred to a clean tube with the addition of 0.1 volume of $5 \mathrm{x}$ CTAB buffer and incubated at $65^{\circ} \mathrm{C}$ for $10 \mathrm{~min}$. After that, an equal volume of chloroform was introduced, stirred on a shaker for $10 \mathrm{~min}$, and centrifuged with the following parameters: $10000 \mathrm{~g}, 10 \mathrm{~min}, 40^{\circ} \mathrm{C}$. The aqueous phase was transferred to clean tubes with the addition of 2 volumes of the buffer for DNA precipitation ( $1 \% \mathrm{CTAB}$, $50 \mathrm{mM}$ Tris- $\mathrm{HCl} \mathrm{pH} 8.0,10 \mathrm{MM}$ EDTA) and incubated at room temperature for 1 hour. It was then centrifuged at 10000 $\mathrm{g}$ for $15 \mathrm{~min}$ at room temperature. Next, the supernatant was collected gently without disturbing the precipitate. The DNA pellet was air-dried for $5 \mathrm{~min}$, dissolved in $2 \mathrm{ml}$ of prewarmed to $60^{\circ} \mathrm{C}$ G-buffer from the Blood \& Cell Culture DNA Mini Kit (Qiagen, USA), and incubated at $60^{\circ} \mathrm{C}$ for $10 \mathrm{~min}$. To the DNA sample in the G-buffer, $4 \mu \mathrm{l}$ of RNase A $(100 \mathrm{mg} / \mathrm{ml}$; 7000 units $/ \mathrm{ml}$; Qiagen) were added and incubated at $37^{\circ} \mathrm{C}$ for $30 \mathrm{~min}$. To this, $25 \mu \mathrm{l}$ of proteinase $\mathrm{K}(>600 \mathrm{mAU} / \mathrm{ml}$; Qiagen) were introduced and incubated at $50^{\circ} \mathrm{C}$ for $40 \mathrm{~min}$. Further, DNA was purified according to the Blood \& Cell Culture DNA Mini Kit (Qiagen) protocol. To the DNA 
elution, 0.7 volume of isopropanol was added, stirred until DNA strands appeared and neatly wrapped them around a glass rod. Then the DNA was transferred to a tube containing a DNA dilution buffer (Evrogen, Russia) and incubated at $50^{\circ} \mathrm{C}$ for $60 \mathrm{~min}$. The DNA quality and concentration were evaluated on a NanoDrop 2000C spectrophotometer (Thermo Fisher Scientific). The DNA concentration was also evaluated on a Qubit 2.0 fluorometer (Life Technologies). The assessment of DNA length and control of the absence of RNA were performed by electrophoresis in a $0.8 \%$ agarose gel (Lonza, Switzerland).

DNA library preparation and sequencing of genomes on the Illumina platform

DNA was fragmented on a S220 ultrasonic homogenizer (Covaris, USA), and $1 \mu \mathrm{g}$ of fragmented DNA was used to prepare the library using the NEBNext Ultra II DNA Library Prep Kit for Illumina (New England Biolabs) according to the manufacturer's protocol with the size selection of adaptorligated DNA of about $600 \mathrm{bp}$. The quality and concentration of DNA libraries were evaluated using a 2100 Bioanalyzer instrument (Agilent Technologies) and a Qubit 2.0 fluorometer (Life Technologies), respectively. The resulting DNA libraries were sequenced on a HiSeq 2500 instrument (Illumina) with a read length of $125+125 \mathrm{bp}$.

DNA library preparation and sequencing of genomes on the Oxford Nanopore platform

To remove short DNA fragments (up to $10 \mathrm{~kb}$ ), the Short Read Eliminator Kit (Circulomics, USA) was used. Then, the DNA sample was diluted to a concentration of $60 \mathrm{ng} / \mathrm{ml}$ in a final volume of $50 \mu \mathrm{l}$ of DNA dilution buffer (Evrogen) and further purified with AMPure XP beads (Beckman Coulter, USA) in a ratio of 1:0.7 (sample:beads).

Preparation of the libraries was performed using the SQKLSK109 Ligation Sequencing Kit (Oxford Nanopore Technologies, UK) for 1D genomic DNA sequencing. Minor modifications were introduced to the recommended protocol for library preparation by increasing the incubation time to 20 min at $20^{\circ} \mathrm{C}$ at the step of combined recovery of DNA using the NEBNext Ultra II End Repair/dA-Tailing Module (New England Biolabs) and NEBNext FFPE DNA Repair Mix (New England Biolabs). Besides, the incubation time at the step of ligation was increased to $60 \mathrm{~min}$. Sequencing was performed on MinION (Oxford Nanopore Technologies) with a FLO-MIN-106 R9.4 flow-cell (Oxford Nanopore Technologies).

\section{Results}

We developed and optimized the method of obtaining pure high-molecular-weight genomic DNA from young poplar leaves needed for Nanopore sequencing. According to this protocol, we extracted the DNA of the following quality: A260/280 ratio laying in the range of 1.77-1.80 and A260/230 being in the range of 1.7-2.3. The obtained proximity of concentration values measured with Qubit and Nanodrop served as an additional criterion of DNA purity. The high DNA length (more than $50 \mathrm{~kb}$ ) and the absence of RNA were confirmed by electrophoresis. After the sequencing of prepared DNA libraries on a MinION instrument, we received $18 \mathrm{~Gb}$ with average $\mathrm{N} 50$ of $25 \mathrm{~kb}$ for each genotype, whereas using a HiSeq 2500 instrument, from 36 to 52 million of pairend $125+125$ reads for male and female plants. The initial genome assembly was performed for the Nanopore data using Shasta, wtdbg2, and Flye tools. Then, these assemblies were consequently polished by Racon (Nanopore data) and Pilon (Illumina data). The Shasta assembler provided the best assembly results: N50 parameter of the assemblies was about $0.5 \mathrm{Mb}$, while the integrity was over $95 \%$ according to BUSCO. The combination of long reads from Nanopore and short high-accuracy reads from Illumina allowed us to achieve a high-quality genome assembly of male and female plants of $P$. x sibirica. Besides, the protocol of pure high-molecular DNA extraction from poplar leaves was developed for further sequencing of material on the Nanopore platform. The analysis of high-quality genomes of male and female Populus $\mathrm{x}$ sibirica species provides one with an opportunity to reveal the genes associated with poplar sex that will facilitate the specification of its sex-determination mechanisms.

\section{Acknowledgment}

This work was funded by RFBR according to the research project 17-29-08036.

\section{References}

[1] Ellis B., Jansson S., Strauss S.H., et al. Why and How Populus Became a "Model Tree". New York, NY: Springer New York, 2010. 